\title{
Radiation Induced Oxidation Reactions of Ferrous Ions: An Agent-based Model
}

\author{
A.L. RIVERA ${ }^{1,2, *}$, A.S. RAMOS-BERNAL ${ }^{1}$, A. NEGRÓN-MENDOZA ${ }^{1}$ \\ ${ }^{1}$ Instituto de Ciencias Nucleares, Universidad Nacional Autónoma de México. \\ Circuito Exterior S/N, Ciudad Universitaria, Coyoacán, 04510 Ciudad de \\ México, México. \\ ${ }^{2}$ Centro de Ciencias de la Complejidad, Universidad Nacional Autónoma de México.

\section{*E-mail: ana.rivera@nucleares.unam.mx}

Published online: August 07, 2017

The Author(s) 2017. This article is published with open access at www .chitkara.edu. in/publications

\begin{abstract}
Chemical Fricke dosimeter in the laboratory can be submitted to gamma radiation at low temperatures to study the evolution of oxidation reactions induced by radiation, a key process to understand the formation of complex molecules. Products generated by the interaction of the different elements under radiation can be determined through a mathematical model that considers chemical reactions as coupled nonlinear ordinary differential equations involving the mass balance of all the species in the reaction. In this paper is implemented an alternative way of solving this system of equations, species' concentrations are calculated through an agent-based model implemented in Python. The model is a modified version of the prey-predator model where each chemical specie involved is considered as an agent that can interact with other specie with known reaction rates leading to production (source terms) and to destruction (sink terms). Here, the radiation is a factor that affects product formation while the bath temperature modifies the reaction speed. This model can reproduce experimental concentrations of products and the consumption of ferrous ions from a laboratory reaction of irradiation of iron salt solutions at 3 different temperatures (dry ice, liquid nitrogen, and room temperature).
\end{abstract}

Keywords: Chemical reactions; Fricke dosimeter; Prey-predator model; Agentbased model.

\section{INTRODUCTION}

In the laboratory is common to use radiation induced chemical oxidation reactions to study the formation of biologically relevant molecules in space

Journal of Nuclear Physics, Material Sciences, Radiation and Applications Vol-5 No-1 August 2017 pp. 15-23 
Rivera, A.L.

Ramos-Bernal, A.S. Negrón-Mendoza, A. bodies due to the energy transfer by radiation [1-6]. Chemical analysis, pulse radiolysis, and competition kinetic methods have been employed experimentally to obtain the large number of rate constants involved in these reactions [7-11]. Mathematical models can follow the dynamics of the radiation induced chemical reactions using a system of coupled nonlinear ordinary differential equations [12-17]. In general, these models cannot be solved exactly because the non-linear coupling of the equations that produces a strong dependence on initial conditions [13], however there are some numerical trials of solution $[12,14,17]$. There is an effort to make computational simulations to understand chemical reactions evolution, using finite-differences [18], Monte-Carlo [19] or agent-based models [20,21]. Continuing the trials with agent based models, here we propose a modified version of [21] considering that each chemical species involved is an agent that can interact with other species with known reaction rates leading to production and destruction terms as in a prey-predator model [22-24]. The radiation is considered as a factor that affects product creation while the bath temperature modifies the reaction speed. This model can reproduce the formation of products and the consumption of ferrous ions in a laboratory experiment.

\section{EXPERIMENTAL SETUP}

Iron salt oxygenated solutions $\left(\mathrm{Fe}_{2} \mathrm{SO}_{4} \cdot 7 \mathrm{H}_{2} \mathrm{O}\right)$ in sulfuric acid were irradiated at a dose rate of $0.93 \mathrm{~Gy} / \mathrm{min}$ in a source of Cobalt 60 (following the setup of Negrón-Mendoza, et al. [2]). Samples were inside Pyrex cells surrounded by a Dewar flask filled with different cooling agents to made a thermal bath at 77 K (liquid Nitrogen), 198 K (dry ice), and 298 K (water at room temperature). After irradiation, the samples were melted, and the temperature was adjusted to room temperature $(298 \mathrm{~K})$. Then, the analysis was performed by UV-Vis spectroscopy at $308 \mathrm{~nm}$ in a Varian Cary100 spectrophotometer (California USA) $\mathrm{Fe}^{3+}$ concentrations were determined and compared with the agentbased model results.

\section{MATHEMATICAL MODEL FOR OXIDATION REACTIONS}

Chemical oxidation reactions for $\mathrm{Fe}^{2+}$ in a Fricke dosimeter are well known $[14,20]$ :

$$
\begin{aligned}
& \mathrm{H}_{2} \mathrm{O} \longrightarrow \mathrm{H}^{\bullet}, \mathrm{e}_{a q}^{-}, \mathrm{OH}, \mathrm{H}_{2} \mathrm{O}_{2}, \mathrm{H}_{3} \mathrm{O}^{+} \\
& \mathrm{H}^{\bullet}+\mathrm{O}_{2} \stackrel{r_{1}}{\longrightarrow} \mathrm{HO}_{2}^{\bullet} \\
& \mathrm{e}_{\mathrm{aq}}-+\mathrm{H}_{3} \mathrm{O}^{+} \stackrel{r_{2}}{\longrightarrow} \mathrm{H}^{\bullet}
\end{aligned}
$$




$$
\begin{aligned}
\mathrm{Fe}^{2+}+\mathrm{OH} \stackrel{r_{3}}{\longrightarrow} \mathrm{Fe}^{3+} \mathrm{OH}^{-} \\
\mathrm{Fe}^{2+}+\mathrm{H}_{2} \mathrm{O}_{2} \stackrel{r_{4}}{\longrightarrow} \mathrm{Fe}^{3+}+\mathrm{OH}+\mathrm{OH}^{-} \\
\mathrm{Fe}^{2+}+\mathrm{HO}_{2} \stackrel{r_{5}}{\longrightarrow} \mathrm{Fe}^{3+}+\mathrm{HO}_{2}^{-} \\
\mathrm{HO}_{2}^{-}+\mathrm{H}^{+} \stackrel{r_{6}}{\longrightarrow} \mathrm{H}_{2} \mathrm{O}_{2} \\
\mathrm{H}_{2} \mathrm{O}_{2} \stackrel{r_{7}}{\longrightarrow} \mathrm{HO}_{2}^{-}+\mathrm{H}^{+}
\end{aligned}
$$

Radiation Induced Oxidation

Reactions of

Ferrous Ions: An

Agent-based Model

In this case, the last chemical reactions involve 14 species $\left(\mathrm{Fe}^{2+}, \mathrm{Fe}^{3+}, \mathrm{H}_{2} \mathrm{O}\right.$, $\mathrm{H}^{\bullet}, \mathrm{H}^{+}, \mathrm{H}_{2}, \mathrm{O}_{2},{ }^{\bullet} \mathrm{OH}, \mathrm{OH}^{-}, \mathrm{H}_{2} \mathrm{O}_{2}, \mathrm{H}_{3} \mathrm{O}^{+}, \mathrm{HO}_{2}^{\bullet}, \mathrm{HO}_{2}^{-}$, and $\mathrm{e}_{\mathrm{aq}}-$ (hydrated electrons), and seven known reaction rates $\left(r_{\mathrm{i}}\right)$. Standard concentrations for the Fricke dosimeter for $\mathrm{Fe}_{2} \mathrm{SO}_{4} \cdot 7 \mathrm{H}_{2} \mathrm{O}$ were employed, with the oxidation of ferrous ions taking place in an acid medium $\left(\mathrm{H}_{2} \mathrm{SO}_{4}\right)$. Initial concentrations were $0.005 \mathrm{M} \mathrm{Fe}^{2+}, 0.012 \mathrm{M} \mathrm{O}_{2}, 0.400 \mathrm{M} \mathrm{H}_{3} \mathrm{O}^{+}$, and the rest of the species had zero concentration (in agreement with [7]). Reaction rates are: $r_{1} \sim 2.0^{*} 10^{10}$ $\mathrm{M}^{-1} \mathrm{~s}^{-1}, r_{2} \sim 2.3 * 10^{10} \mathrm{M}^{-1} \mathrm{~s}^{-1}, r_{3} \sim 3.0^{*} 10^{8} \mathrm{M}^{-1} \mathrm{~s}^{-1}, r_{4} \sim 50 \mathrm{M}^{-1} \mathrm{~s}^{-1}, r_{5} \sim r_{6} \sim 7.3 * 10^{5}$ $\mathrm{M}^{-1} \mathrm{~s}^{-1}$, and $r_{7} \sim 10^{5} \mathrm{M}^{-1} \mathrm{~s}^{-1}$.

Kinetics of the radiation induced chemical reactions in a thermal bath (eqs. 1) involves the mass balance for each of the 14 species. This balance, assuming that is due with the interaction between pairs, can be described by coupled nonlinear differential equations considering external source (radiation and bath temperature), production (source terms) and destruction (sink terms), as proposed on a previous work $[15,19]$ :

$$
\frac{d N_{i}(t)}{d t}=f_{i}+\sum_{j} \sum_{k} r_{j, k}^{(i)} N_{j}(t) N_{k}(t)-N_{i}(t) \sum_{m} r_{i, m}^{(i)} N_{m}(t),
$$

where denotes the molar concentration of the -species at time $t, f_{i}$ is an external source, and $r_{j, k}^{(i)}$ denotes the reaction rate constant for the two-reactive species $j, k$ that produce the specie $(i)$.

Here, we consider that the external source term $f_{i}$, is the number of molecules of the $i$-species produced in the radiolysis process induced by the gamma radiation absorbed in the medium at the thermal bath temperature $T$. As first approximation, we will take it given as a Maxwell-Boltzmann distribution for temperature, and as a linear function of the radiation dose intensity $I_{d}$, modifying the expression of previous publications $[15,19]$ :

$$
f_{i}\left(I_{d}, T\right)=\frac{6.2 \times 10^{11}}{3.6 N_{A}} \frac{M_{i}}{M_{H_{2} O}} G_{i} e-\frac{M_{i}}{2 N_{A} k T} I_{d},
$$


Rivera, A.L.

Ramos-Bernal, A.S. Negrón-Mendoza, A. where $M_{i}$ is the molecular mass of the species $i, M_{H 2 O}$ is the molecular mass of water $(18.02 \mathrm{~g} / \mathrm{mol}), N_{A}$ is the Avogadro number $\left(6.022 \times 10^{23} \mathrm{~mol}\right)$, is the Boltzmann constant $\left(1.38 \times 10^{-23} \mathrm{~m}^{2} \mathrm{kgs}^{-2} \mathrm{~K}^{-1}\right)$, and $G_{i}$ a constant, the radiochemical yield that gives the number of species $i$ produced when $100 \mathrm{eV}$ are absorbed by the system. For the case of $\mathrm{Fe}^{3+}, G_{i}$ is 15.6 molecules.

Chemical reactions described by the system of eqs. (1) can be rewritten as a system of nonlinear differential equations using eqs. $(2,3)$, one for each species involved:

$$
\begin{aligned}
& \frac{d N_{\mathrm{H}^{\bullet}}(t)}{d t}=f_{\mathrm{H}^{\bullet}}\left(I_{d}, T\right)+r_{2} N_{e_{a q}^{-}}(t) N_{\mathrm{H}_{3} \mathrm{O}^{+}}(t)-r_{1} N_{\mathrm{O}_{2}}(t) N_{\mathrm{H}^{\bullet}}(t), \\
& \frac{d N_{\mathrm{H}^{+}}(t)}{d t}=f_{\mathrm{H}^{+}}\left(I_{d}, T\right)+r_{7} N_{\mathrm{H}_{2} \mathrm{O}_{2}}(t)-r_{6} N_{\mathrm{H}^{+}}(t) N_{\mathrm{HO}_{2}^{-}}(t), \\
& \frac{d N_{\mathrm{O}_{2}}(t)}{d t}=f_{\mathrm{O}_{2}}\left(I_{d}, T\right)-r_{1} N_{\mathrm{O}_{2}}(t) N_{\mathrm{H}^{*}}(t), \\
& \frac{d N_{\mathrm{HO}_{2}^{*}}(t)}{d t}=f_{\mathrm{HO}_{2}^{*}}\left(I_{d}, T\right)+r_{1} N_{\mathrm{O}_{2}}(t) N_{\mathrm{H}^{\bullet}}(t)-r_{5} N_{\mathrm{HO}_{2}^{\bullet}}(t) N_{\mathrm{Fe}^{2+}}(t), \\
& \frac{d N_{\mathrm{H}_{3} \mathrm{O}^{+}}(t)}{d t}=f_{\mathrm{H}_{3} \mathrm{O}^{+}}\left(I_{d}, T\right)-r_{2} N_{e_{a q}^{-}}(t) N_{\mathrm{H}_{3} \mathrm{O}^{+}}(t), \\
& \frac{d N_{\mathrm{OH}}(t)}{d t}=f_{\mathrm{OH}}\left(I_{d}, T\right)+r_{4} N_{\mathrm{Fe}^{2+}}(t) N_{\mathrm{H}_{2} \mathrm{O}_{2}}(t)-r_{3} N_{\mathrm{Fe}^{2+}}(t) N_{\mathrm{OH}}(t), \\
& \frac{d N_{\mathrm{OH}^{-}}(t)}{d t}=f_{\mathrm{OH}^{-}}\left(I_{d}, T\right)+r_{3} N_{\mathrm{Fe}^{2+}}(t) N_{\mathrm{OH}}(t)+r_{4} N_{\mathrm{Fe}^{2+}}(t) N_{\mathrm{H}_{2} \mathrm{O}_{2}}(t), \\
& \frac{d N_{\mathrm{H}_{2} \mathrm{O}_{2}}(t)}{d t}=f_{\mathrm{H}_{2} \mathrm{O}_{2}}\left(I_{d}, T\right)+r_{6} N_{\mathrm{H}^{+}}(t) N_{\mathrm{HO}_{2}^{-}}(t)-r_{4} N_{\mathrm{Fe}^{2+}}(t) N_{\mathrm{H}_{2} \mathrm{O}_{2}}(t) \\
& -r_{7} N_{\mathrm{H}_{2} \mathrm{O}_{2}}(t) \text {, } \\
& \frac{d N_{\mathrm{HO}_{2}^{-}}(t)}{d t}=f_{\mathrm{HO}_{2}^{-}}\left(I_{d}, T\right)+r_{5} N_{\mathrm{HO}_{2}}(t) N_{\mathrm{Fe}^{2+}}(t)-r_{6} N_{\mathrm{H}^{+}}(t) N_{\mathrm{HO}_{2}^{-}}(t), \\
& \frac{d N_{\mathrm{Fe}^{2+}}(t)}{d t}=f_{\mathrm{Fe}^{2+}}\left(I_{d}, T\right)-r_{3} N_{\mathrm{OH}}(t) N_{\mathrm{Fe}^{2+}}(t)-r_{4} N_{\mathrm{H}_{2} \mathrm{O}_{2}}(t) N_{\mathrm{Fe}^{2+}}(t) \\
& -r_{5} N_{\mathrm{HO}_{2}^{*}}(t) N_{\mathrm{Fe}^{2+}}(t),
\end{aligned}
$$




$$
\begin{aligned}
\frac{d N_{\mathrm{Fe}^{3+}}(t)}{d t}=f_{\mathrm{Fe}^{3+}}\left(I_{d}, T\right)+r_{3} N_{\mathrm{OH}}(t) N_{\mathrm{Fe}^{2+}}(t) & +r_{4} N_{\mathrm{H}_{2} \mathrm{O}_{2}}(t) N_{\mathrm{Fe}^{2+}}(t) \\
& +r_{5} N_{\mathrm{HO}_{2}}(t) N_{\mathrm{Fe}^{2+}}(t),
\end{aligned}
$$

This system of coupled nonlinear differential equations can be solved by finite difference methods as Sanchez-Mejorada, et al. [14] or Cruz-Castañeda et al [15] done. The problem with this solution scheme is that the system has a strong dependence on initial conditions and to guaranty convergence some extra parameters need to be added to the model. Moreover, there are some computational troubles due to the very wide range of the reaction rates that differ more than 8 orders of magnitude.

\section{AGENT-BASED MODEL}

An alternative numerical solution for eqs. (4) can be found using an agentbased model with sources and sinks that can be interpreted as prey and predators. The agents are in a thermal bath at temperature $\mathrm{T}$ under a gamma radiation immerses in water with free electrons. We made a Python program to follow the molar concentrations of each species. The code initializes a region of 1,024 × 1,024 pixels, with 40,000 randomly allocated points associated with $\mathrm{H}_{3} \mathrm{O}^{+}, 1,200$ points of $\mathrm{O}_{2}$, and 500 of $\mathrm{Fe}^{2+}$. In contrast with a previous work [20], for every step, the specie of each point of the grid moves with a velocity produced by the thermal agitation of the molecules using a MaxwellBoltzmann distribution:

$$
v_{i}=\sqrt{\frac{3 k N_{A} T}{M_{i}}} .
$$

In each grid point is one of the species involved; arrows correspond to velocity of the specie given by eq. 5 . Two points react in agreement with eqs. (1) when they are in the same point.

After the specie moves, if in the grid point there are two species that can react in accordance to eqs. (1), the reaction takes place consuming the "prey" elements by the "predators" produced (see Figure 1). If the velocity of a given specie takes it out of the border of the network, the specie stays in its actual position. The rate of reaction is determined by . After actualizing the entire net, the procedure is repeated until the steady-state is reached. In the program, we can follow the molar concentration of each species as a function of time (see Figure 2). In Figure 3, the molar concentration of $\mathrm{Fe}^{3+}$ is plotted and compared with experimental data.
Radiation Induced Oxidation Reactions of Ferrous Ions: An Agent-based Model

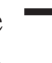


Rivera, A.L.

Ramos-Bernal, A.S.

Negrón-Mendoza, A.

Figure 1: Agent based model for Fricke dosimeter.

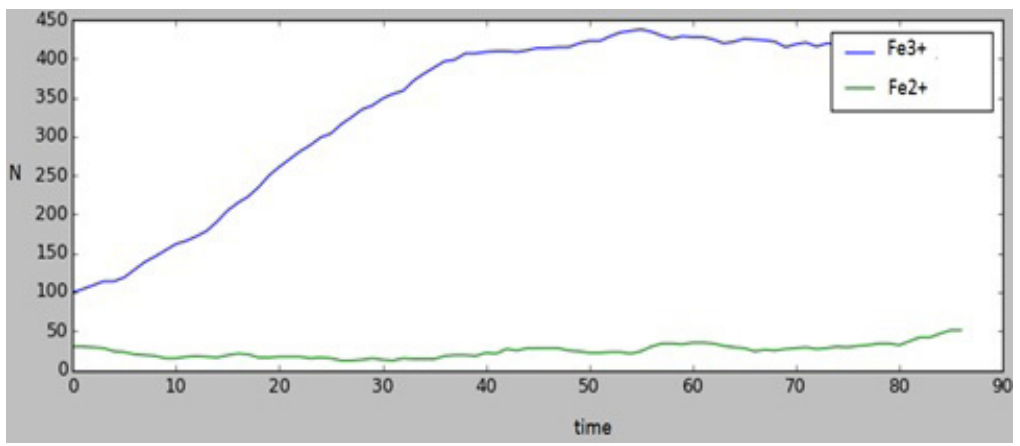

Figure 2:. Molar concentrations of $\mathrm{Fe}^{2+}$ and $\mathrm{Fe}^{3+}$ as function of time.

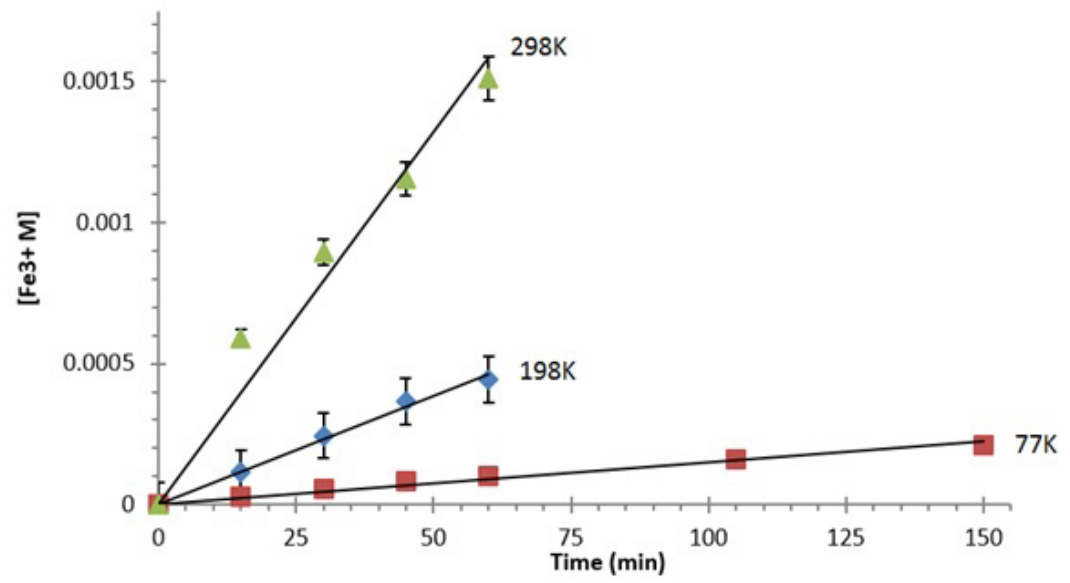

Figure 3: Molar concentration of $\mathrm{Fe}^{3+}$. The points correspond to experimental values and the continuous line to the agent-based results, at $77 \mathrm{~K}$ (liquid Nitrogen), $198 \mathrm{~K}$ (dry ice), and $298 \mathrm{~K}$ (water at room temperature). 


\section{CONCLUSION}

In this paper we described the kinetics of the oxidation of ferrous ions induced by gamma radiation in a Fricke dosimeter in a thermal bath at $77 \mathrm{~K}$ (liquid Nitrogen), $198 \mathrm{~K}$ (dry ice), and $298 \mathrm{~K}$ (water at room temperature). Instead of using traditional methods to solve the system of coupled non-linear ordinary differential equations that describe the mass balance of all the species involved (eqs. 4), here we implemented an original agent based model in Python that includes in eqs. (4) all of the reaction rates as source and sink terms (like in a prey-predator model) with an external term due to the radiation induced product formation and the reaction velocity given by a Maxwell-Boltzmann distribution. The agreement of the results from our agent-based model with the experimental results suggests that our numerical model is reliable for analyzing the kinetics of chemical oxidation reactions induced by radiation in a thermal bath. This model can be used to understand the kinetics of reactions induced by radiation when the temperature of the irradiated medium decreases below the freezing point. Further studies and experiments are needed to validate and establish the applicability of our model.

\section{ACKNOWLEDGMENTS}

Partial financial support was given by UNAM under project PAPIIT IN106215, and CONACYT grant 168579.

\section{REFERENCES}

[1] Negrón-Mendoza, A, \& Ponnamperuma, C. (1982). Prebiotic formation of higher molecular weight compounds from the photolysis of aqueous acetic acid. Photochemistry and Photobiology, 36(5), 595-597, doi: 10.1111/j.17511097.1982.tb04421.x.

[2] Draganić, Z.D., Vujošević, S.I., Negrón-Mendoza, A., Azamar, J.A., \& Draganić, I.G. (1985). Radiation chemistry of a multicomponent aqueous system relevant to chemistry of cometary nuclei. Journal of molecular evolution, 22(2), 175-187, doi: 10.1007/BF02101695.

[3] Negrón-Mendoza, A., Albarran, G., Ramos, S., \& Chacon, E. (1995). Some aspects of laboratory cometary models. Journal of Biological Physics, 20(1), 71-76, doi: 10.1007/BF00700422.

[4] Ehrenfreund, P., \& Charnley, S. B. (2000). Organic molecules in the interstellar medium, comets, and meteorites: A Voyage from Dark Clouds to the Early Earth. Annual Review of Astronomy and Astrophysics, 38, 427-483, doi: 10.1146/ annurev.astro.38.1.427.
Radiation Induced Oxidation

Reactions of

Ferrous Ions: An

Agent-based Model

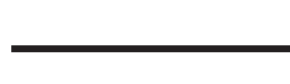


Rivera, A.L.

Ramos-Bernal, A.S. Negrón-Mendoza, A.
[5] Colín-García, M., Negrón-Mendoza, A., \& Ramos-Bernal, S. (2009). Organic material formed from gamma irradiation of frozen $\mathrm{HCN}$ as a cometary ice analog: implication to the origin of life. International Journal of Astrobiology, 9 , 279-288.

[6] Taquet,V.,Wirström,E.S.,\& Charnley,S.B.(2016).Formation and recondensation of complex organic molecules during protostellar luminosity outbursts. The Astrophysical Journal, 821(1), 46, doi: 10.3847/0004-637X/821/1/46.

[7] O’Donnell, J.H.O., \& Sangster, D.F. (1970). Principles of Radiation Chemistry. Elsevier, New York.

[8] Schmidt, K.H. (1972). Electrical conductivity techniques for studying the kinetics of radiation-induced chemical reactions in aqueous solutions. International Journal for Radiation Physics and Chemistry, 4(4), 439-468, doi: 10.1016/00207055(72)90020-4.

[9] Castillo, S. Negrón-Mendoza, A., Draganic, Z.D., \& Draganic, I.G. (1985). The radiolysis of aqueous solutions of malic acid. Radiation Physics and Chemistry, 26(4), 437-443, doi: 10.1016/0146-5724(85)90232-8.

[10] Draganic, Z.D., Negrón-Mendoza, A., Navarro-González, R., \& Vujosevic, S.I. (1987). The presence of polymeric material in radiolyzed aqueous solution of ammonium bicarbonate. International Journal of Radiation Applications and Instrumentation. Part C. Radiation Physics and Chemistry, 30(4), 229-231, doi: 10.1016/1359-0197(87)90126-3.

[11] Bjergbakke, E., Draganić, Z. D., Sehested, K., \& Draganić, I. G. (1989). Radiolytic products in waters. Radiochimica Acta, 48(1), 73-78, doi: 10.1524/ ract.1989.48.12.73.

[12] Keene, J.P. (1960). Kinetics of radiation-induced chemical reactions. Nature 188(4753), 843-844, doi: 10.1038/188843b0.

[13] Malchow, H., \& Petrovskii, S.V. (2002). Dynamical stabilization of an unstable equilibrium in chemical and biological systems. Mathematical and Computer Modelling, 36(3), 307-319.

[14] Sánchez-Mejorada, G., Frías, D., Negrón-Mendoza, A., \& Ramos-Bernal, S. (2008). A comparison between experimental results and a mathematical model of the oxidation reactions induced by radiation of ferrous ions. Radiation Measurements, 43(2), 287-290, doi: 10.1016/j.radmeas.2007.11.038.

[15] Cruz-Castañeda, J., Negrón-Mendoza, A., Frías, D., Colín-García, M., Heredia, A., et al. (2015). Chemical evolution studies: The radiolysis and thermal decomposition of malonic acid. Journal of Radioanalytical and Nuclear Chemistry, 304(1), 219-225, doi: 10.1007/s10967-014-3711-z.

[16] Semenov, S.N., Kraft, L.J., Ainla, A., Zhao, M., Baghbanzadeh, M., Campbell, V.E., et al. (2016). Autocatalytic, bistable, oscillatory networks of biologically relevant organic reactions. Nature, 537(7622), 656-660, doi: 10.1038/ nature19776. 
[17] Morii, Y., Terashima, H., Koshi, M., Shimizu, T., \& Shima, E. (2016). ERENA: A fast and robust Jacobian-free integration method for ordinary differential equations of chemical kinetics. Journal of Computational Physics, 322, 547-558, doi: 10.1016/j.jcp.2016.06.022.

[18] Garvie, M.R. (2007). Finite-Difference schemes for reaction-diffusion equations modeling Predator-Prey interactions in MATLAB. Bulletin of mathematical biology 69(3), 931-956, doi: 10.1007/s11538-006-9062-3.

[19] Zhdanov, V.P. (2002). Monte Carlo simulations of oscillations, chaos and pattern formation in heterogeneous catalytic reactions. Surface Science Reports, 45(7), 231-326, doi: 10.1016/S0167-5729(01)00023-1.

[20] Rivera, A.L., Negrón-Mendoza, A., \& Ramos-Bernal, S. (2016). “Agent-based model of oxidation reactions of ferrous ions". Journal of Nuclear Physics, Material Sciences, Radiation and Applications 4(1), 149-157, doi: 10.15415/ jnp.2016.41015.

[21] Ausloos, M., Dawid, H., \& Merlone, U. (2015). Spatial interactions in agentbased modeling. In Complexity and Geographical Economics (pp. 353-377). Springer International Publishing.

[22] Berryman, A.A. (1992). The origin and evolution of predator-prey theory. Ecology 73(5), 1530-1535, doi: 10.2307/1940005.

[23] Pascual, M. (1993). Diffusion-induced chaos in a spatial predator-prey system. Proceedings of the Royal Society of London B, 251(1330), 1-7, doi: 10.1098/ rspb.1993.0001.

[24] Pal, D., \& Mahapatra, G.S. (2016). Dynamic behavior of a predator-prey system of combined harvesting with interval-valued rate parameters. Nonlinear Dynamics, 83, 2113-2123, doi: 10.1007/s11071-015-2469-3.
Radiation Induced Oxidation Reactions of Ferrous Ions: An Agent-based Model 